\title{
An increase in circulating levels of branched-chain amino acids during hemodialysis with regard to protein breakdown: three case reports
}

\author{
Masako Fujiwara ${ }^{1,2^{*}}$ (D) Itiro Ando ${ }^{1,2}$, You Shishido ${ }^{3}$, Yutaka Imai $^{2}$ and Hiroyuki Terawaki $^{1}$
}

\begin{abstract}
Background: Hemodialysis (HD) is a protein catabolic event. However, the amino acid (AA) kinetics during HD sessions involved in protein breakdown have not been well investigated in patients with and without diabetes mellitus (DM).

Case presentation: Three patients (two patients with DM and one patient without DM) underwent fasting HD. Plasma levels of branched-chain AAs (BCAA; leucine, isoleucine, and valine), major non-essential AAs (alanine and glutamine, including glutamate), insulin, and ketone bodies were measured every hour during each HD session. After the start of the HD session, the plasma levels of insulin and all BCAAs dropped simultaneously. There was a significant subsequent increase in the plasma level of leucine and isoleucine levels, while valine levels remained constant. However, the recovery in levels of BCAAs during HD indicated a profound amount of BCAAs entering the blood from body tissues such as muscles. BCAAs may have surpassed their removal by HD. Ketone body levels increased continuously from the start of the sessions and reached high values in patients with DM. Synchronous changes in insulin depletion and an increase in the levels of ketone bodies may indicate disruption of energy metabolism.
\end{abstract}

Conclusions: This is the first report to demonstrate the time course of the changes in circulating levels of BCAAs and related metabolites in energy homeostasis during HD. An increase in BCAA levels during HD was found to be due to their transfer from the body tissue which suggested protein breakdown.

Keywords: Branched-chain amino acids, Cell starvation, Diabetes mellitus, Glucose transporter, Hemodialysis, Insulin, Ketone bodies, Protein breakdown

\section{Background}

Hemodialysis (HD) involves protein catabolism accompanied by muscle proteolysis [1]. HD is associated with an increase in amino acid (AA) loss from blood, which induces muscle protein breakdown $[2,3]$. However, detailed AA kinetics and mechanisms involved in skeletal

*Correspondence: m-fujiwara@med.teikyo-u.ac.jp

1 Department of Internal Medicine, Nephrology, Teikyo University Chiba Medical Center, 3426-3, Anesaki, Ichihara, Chiba 299-0111, Japan

Full list of author information is available at the end of the article protein breakdown during HD have not been investigated in patients with and without diabetes mellitus (DM).

In DM patients with various pathophysiological conditions, including insulin resistance, response to HD sessions regarding energy metabolism and AA behaviors differ from the response seen in non-DM patients [4-6]. In our previous study, we found that patients with DM often experienced serious "cell starvation" during HD [6]. The rapid removal of insulin from the blood by HD caused cell starvation (HD starvation), which accompanies an increase in the levels of ketone bodies due 
to low glucose levels within the cell, even though there was sufficient glucose in the blood. Meanwhile, the AA behavior during the HD session has not been sufficiently examined.

Thus, we aimed to investigate the circulating AA behaviors and profiles of insulin and ketone bodies during HD sessions in two patients with DM and one patient without DM.

\section{Case presentation and methods}

The characteristics of the three patients (Patients 1 and 2 with DM, and Patient 3 without DM) are shown in Table 1. Among them, two patients (Cases 2 and 3) underwent online pre-dilution hemodiafiltration (HDF) as a regular treatment. All patients were in a stable condition and showed no signs of malnutrition. In all cases, the glucose concentration of the dialysate was at $100 \mathrm{mg} / \mathrm{dL}$. They all had breakfast before HD or HDF sessions and received no exogenous AA during
HD sessions. In Case 1 and 2, they refrained from insulin injection during HD session: they underwent insulin injection with the intake of first meal after HD session. In three (for two DM cases) and two (for a non-DM case) sessions, plasma samples were collected from the blood tubing at the arterial site every hour during each HD session using a method reported in a previous study [7]. For Case 2 and 3, we were not allowed to collect blood specimen because of anemic tendency in these patients. Therefore, we used dialysate as a surrogate of plasma to measure $\mathrm{KB}$ levels as mentioned below.

Plasma levels of the following molecules were quantified from the samples collected: major components of non-essential AAs (NEAAs) (alanine [Ala], glutamine, and glutamate), three branched-chain AAs (BCAAs) (leucine [Leu], isoleucine [Ile], and valine [Val]), immunoreactive insulin (IRI), and ketone bodies (KB; including 3-hydroxy butyrate and acetoacetate). As glutamine

Table 1 Characteristics of study patients

\begin{tabular}{|c|c|c|c|}
\hline & Case 1 & Case 2 & Case 3 \\
\hline Primary CKD & Rapidly progressive glomerulonephritis & Diabetic kidney disease & $\begin{array}{l}\text { Chronic } \\
\text { glomerulone- } \\
\text { phritis }\end{array}$ \\
\hline Sex & $\mathrm{m}$ & $\mathrm{m}$ & f \\
\hline Age (years) & 76 & 64 & 72 \\
\hline Dialysis vintage (years) & 12 & 1 & 11 \\
\hline $\operatorname{BMI}\left(\mathrm{kg} / \mathrm{m}^{2}\right)$ & 26 & 24 & 25 \\
\hline Weight (kg) & 71 & 71 & 56.3 \\
\hline HD/HDF (h) & $H D, 4 h$ & HDFpre, $5 \mathrm{~h}$ & HDFpre, $5 \mathrm{~h}$ \\
\hline Creatinine $(\mathrm{mg} / \mathrm{dL})$ & 11.3 & 9.7 & 9.4 \\
\hline Albumin $(\mathrm{g} / \mathrm{dL})$ & 3.4 & 3.8 & 3.7 \\
\hline Hemoglobin (g/dL) & 11 & 11 & 11 \\
\hline Hematocrit (\%) & 32 & 31 & 32 \\
\hline Glucose (mg/dL) & 125 & 163 & 98 \\
\hline $\mathrm{CRP}(\mathrm{ng} / \mathrm{mL})$ & 0.04 & 0.01 & 0.1 \\
\hline Phosphate (mg/dL) & 5.5 & 4.8 & 4.2 \\
\hline Potassium (mEq/L) & 5.2 & 5.1 & 4.3 \\
\hline GA (\%) & 12.2 & 18.7 & - \\
\hline GNRI & 92 & 100 & 96 \\
\hline nPCR (g/kg/day) & 0.91 & 1.0 & 1.1 \\
\hline $\mathrm{Kt} / \mathrm{N}$ & 1.65 & 1.39 & 2.1 \\
\hline Raid acting insulin (E) & $3-3-3$ & $5-5-4$ & - \\
\hline Dialyzer (material) & $N V-21 U(P S)$ & $\mathrm{ABH}-21 \mathrm{P}(\mathrm{PS})$ & $\mathrm{ABH}-18 \mathrm{P}(\mathrm{PS})$ \\
\hline Substitution fluid volume (L) & - & 40 & 40 \\
\hline Dialysate flow rate (ml/min) & 500 & 500 & 500 \\
\hline Blood flow rate (ml/min) & 240 & 300 & 240 \\
\hline
\end{tabular}

$C K D$ chronic kidney disease, $B M I$ body mass index, $H D$ hemodialysis, HDF hemodiafiltration, HDFpre pre-dilution hemodiafiltration, $C R P C$-reactive protein, $n P C R$ normalized protein catabolic rate, GA glycated albumin, GNRI geriatric nutritional risk index; The dialyzers were polysulfone (PS) (Toray Medical Co., Ltd, Tokyo Japan).: Data were averaged over three or two sessions 

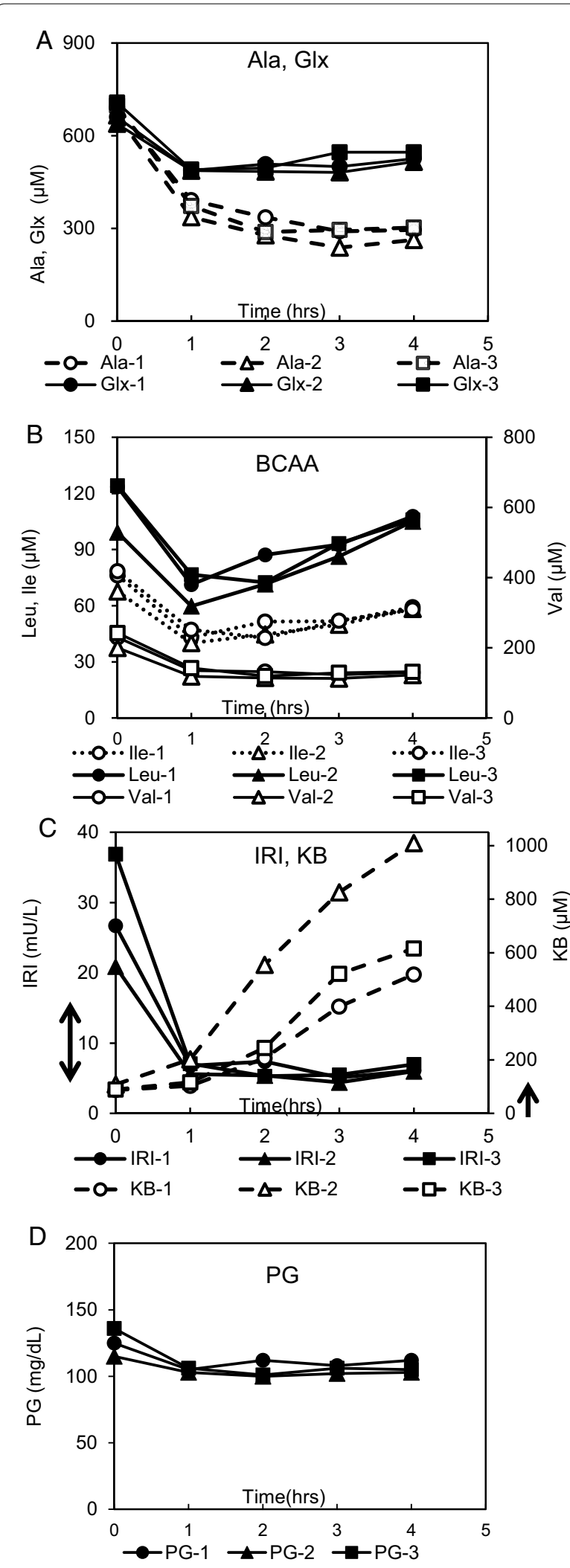

Fig. 1 Plasma levels of metabolites during sessions. Case 1 (DM patient, 3 sessions in 4-h HD). In each panel of Figs. 1, 2, and 3, A Alanine (Ala) and glutamine including glutamate (GlX). B Leucine (Leu), isoleucine (lle) and valine (Val). C Immunoreactive insulin (IRI) and ketone bodies (KB). D Plasma glucose (PG). In C, the arrows along the left and right axis indicate the normal ranges of $I R I$ and $K B$, respectively. The levels of $\mathrm{KB}$ in Fig. $2 \mathrm{C}$ and $2 \mathrm{C}$ were determined at $15 \mathrm{~min}, 30 \mathrm{~min}, 1 \mathrm{~h}, 2 \mathrm{~h}, 3 \mathrm{~h}$ and $4.5 \mathrm{~h}$ after the initiation of dialysis (see text)

is easily converted to glutamate in normal laboratory settings [8], the sum of glutamine and glutamate levels was evaluated as glutamine-glutamate (Glx) levels. The levels of IRI, AA, and glucose were determined using CLIA, HPLC, and enzymatic methods, respectively. The KB levels for Case 1 were determined using an enzymatic method (LSI Medience Corp., Tokyo, Japan). The KB levels for Cases 2 and 3 were measured using dialysate collected at $15 \mathrm{~min}, 30 \mathrm{~min}, 1 \mathrm{~h}, 2 \mathrm{~h}, 3 \mathrm{~h}$, and $4.5 \mathrm{~h}$ after the start of the sessions for determination by ${ }^{1} \mathrm{H}-\mathrm{NMR}$ spectroscopy (ECA, $600 \mathrm{MHz}$, JEOL. Co. LTD., Tokyo, Japan) and converted to plasma levels [5, $6,9,10]$.

This study was approved by the Ethical Committee of Teikyo University (TU-20-194), and informed consent was obtained from all the patients.

\section{Case 1: DM, male patient, three sessions of 4-h HD}

Figure 1A shows the time course of changes in the levels of Ala and Glx. The levels of these AAs decreased thorough removal from the plasma during the first hour of HD. Subsequently, the levels of Glx and Ala remained almost constant toward the end of the sessions. This behavior was similar in Case 2 (Fig. 2A) and Case 3 (Fig. 3A). However, the changes in BCAAs were different from those in the NEAAs (Fig. 1B). Although the levels of BCAAs decreased due to removal during the first hour of HD sessions, dramatic increases in the levels of Leu were observed, and the levels ultimately almost recovered along with the simultaneous increase in Ile levels. Val levels remained constant and showed no decrease. Figure $1 C$ shows the time-course changes in the IRI and KB levels. The IRI levels exhibited a sharp decrease in the first hour of HD. Thereafter, IRI values remained at the lower limit of the normal range (arrow along the left axis) [11]. In contrast, the KB levels continuously and dramatically increased, reaching a remarkably high value of 600 to1000 $\mu \mathrm{M}$. These levels were far higher than the upper limit of the normal range (arrow along the right axis). The time course behaviors of the seven metabolite levels were reproduced in the three sessions. 

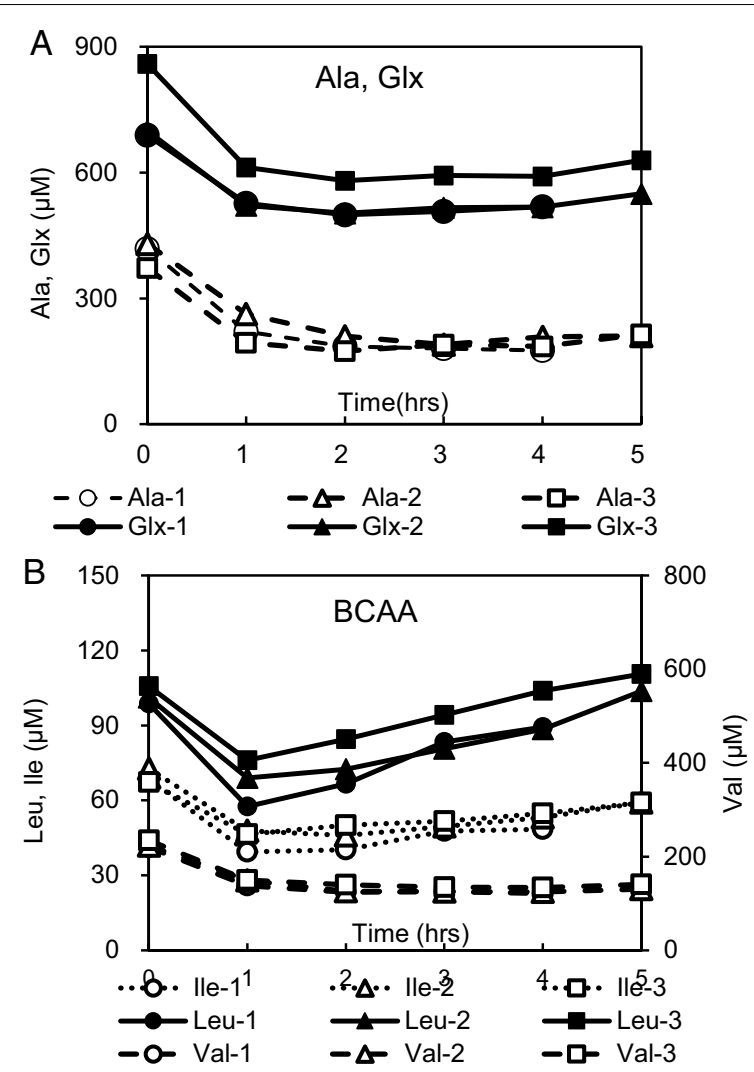

C 40
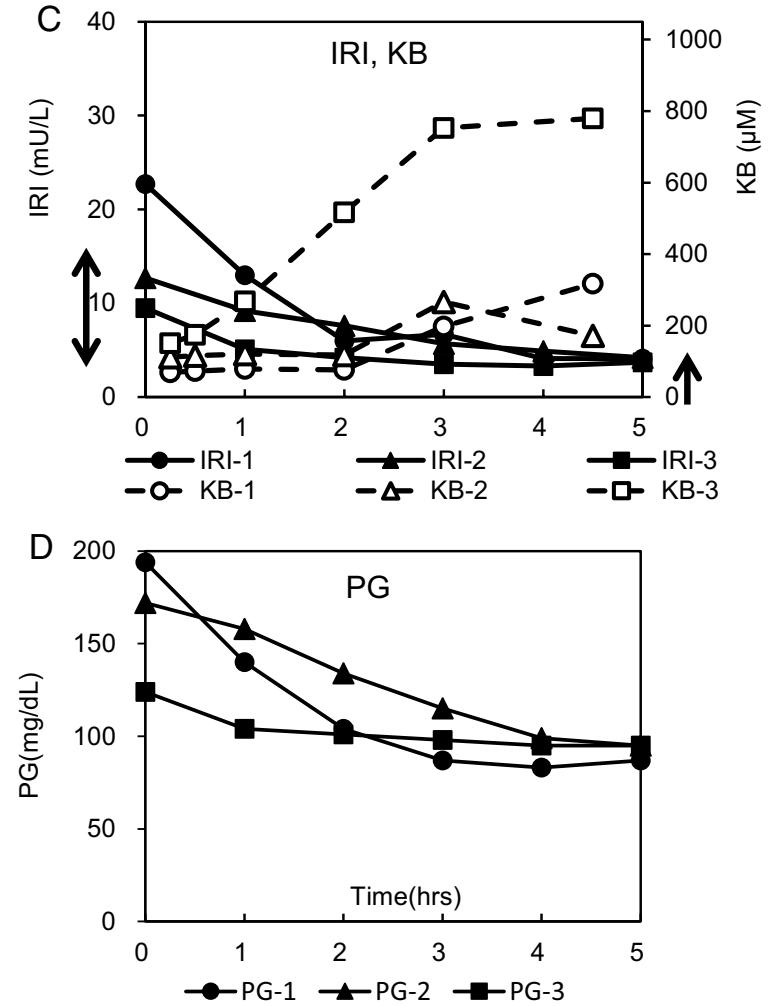

Fig. 2 Plasma levels of metabolites during sessions. Case 2 (DM patient, 3 sessions in 5-h HDF)
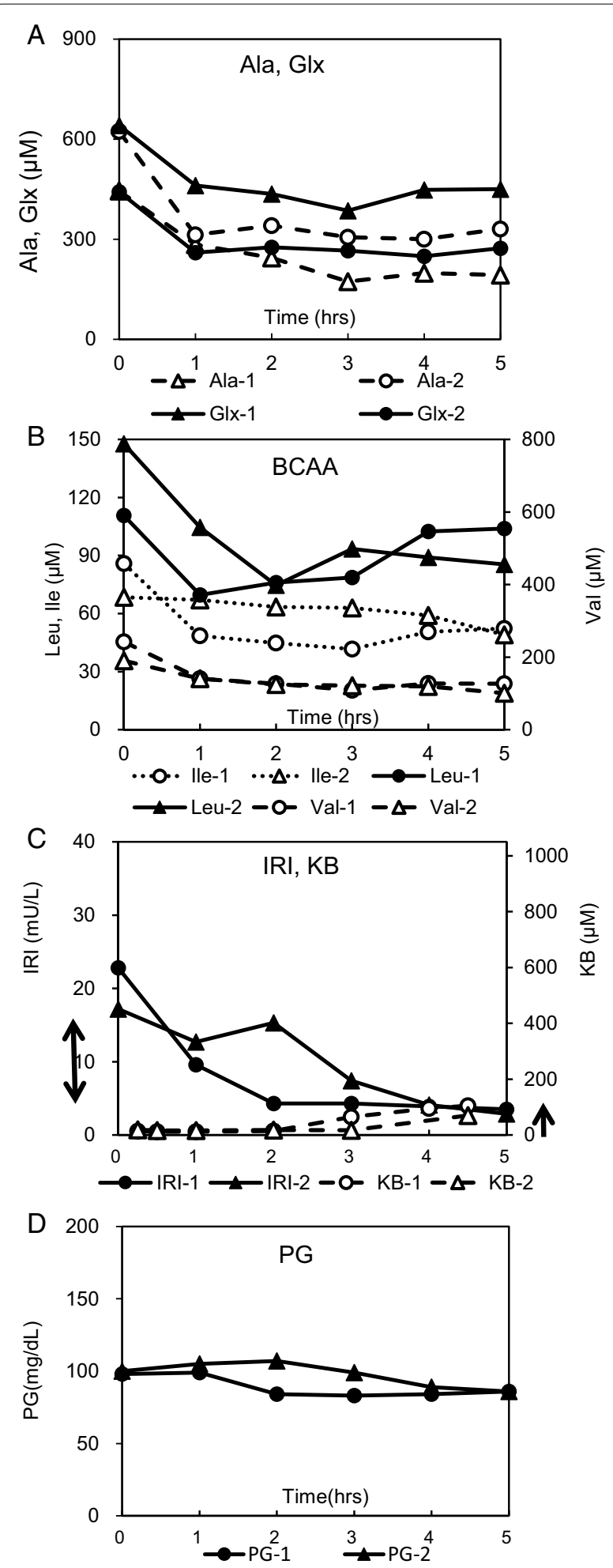

Fig. 3 Plasma levels of metabolites during sessions. Case 3 (non-DM patient, two sessions in 5-h HDF) 
Figure 1D shows the time-course change in the level of plasma glucose (PG), which was almost constant. The PG levels were almost the same in Case 2 (Fig. 2D) and Case 3 (Fig. 3D).

\section{Case 2: DM, male patient, three sessions of 5-h HDF}

Figure $2 \mathrm{~B}$ shows the time changes of BCAAs, which were also very similar to those in Fig. 1B. The dramatic increase in Leu levels was also shown directly at the end of 5-h sessions, and the final levels recovered to slightly above than the initial levels. Figure $2 \mathrm{C}$ illustrates the time changes in the levels of IRI and KB, in which whole behaviors were similar through the sessions with a slower decrease in IRI levels than those presented in Fig. 1C. $\mathrm{KB}$ levels increased with time, and toward the end of the sessions, they reached levels above the normal range. Among the three sessions in Case 2, five types of AAs showed highly reproducible time-course behaviors, and the time-course changes in the levels of IRI and KB were reproduced.

\section{Case 3: Non-DM, female patient, two sessions of 5-h HDF} Figure $3 \mathrm{~B}$ shows the time changes in the levels of BCAAs; those of Leu and Ile somewhat differed from those presented in Figs. $1 \mathrm{~B}$ and $2 \mathrm{~B}$. The levels of Leu and Ile decreased during the first or second hour from the start of the sessions and subsequently exhibited some fluctuations in the time course, and the final levels that recovered were lower than those in Figs. 1B and 2B. However, the changes in the levels of Val were remarkably similar to those in Figs. $1 \mathrm{~B}$ and Fig. 2B. Figure $3 \mathrm{C}$ represents the time changes in the levels of IRI and $K B$, in which IRI decreased during the second hour from the start of the sessions, similar to those seen in Fig. 2C. The changes in the levels of $\mathrm{KB}$ in this case did not increase as much as in Figs. $1 \mathrm{C}$ and $2 \mathrm{C}$, but remained near the upper normal range. In this case, the reproducibility of each metabolite between the two sessions was also confirmed, as long as behaviors in the levels of Leu and Ile had a little time deviation.

\section{Discussion}

The results of present study were summarized as follows:

(A) BCAA levels decreased during the first or second hours and subsequently did not decrease; however, they were remarkably elevated toward the end of the sessions and the profiles were prominent in the patients with DM than the patient without DM.
(B) The levels of Ala and Glx decreased during the first hour and subsequently remained constant.

(C) IRI levels declined sharply between the first and second hours in all patients, and KB levels dramatically increased after the first hour in patients with DM.

(D) PG levels were almost constant.

In general, plasma levels of small molecules without marked derivation (e.g., urea nitrogen) decrease monotonously. Molecular weights of AAs and KB are small and exist in free in serum, and thus, those should be removed by diffusion during hemodialysis treatment. Nevertheless, in this case report, AAs were not decreasing monotonously $(\mathrm{A}, \mathrm{B})$ and $\mathrm{KB}$ was increasing during hemodialysis $(C)$. We suppose that such behavior of plasma $A A s$ and $K B$ levels during HD sessions suggests that large amounts of AAs and KB were derived from cells and such derivation from cells surpasses removal via dialyzer.

Because BCAA is an essential AA, which cannot be biosynthesized, and the patients had no exogenous AA supply during the sessions, these increases are supposed to originate from the free AA pool derived from muscle protein degradation. From our preliminary observation, the two DM patients were more evident with their reproduced time course of changes, while the non-DM patient exhibited blunt behaviors of increase in Leu and Ile levels compared to the DM patients. These final levels did not reach their initial levels.

Ala and Glx constitute the two most important nitrogen carriers released from muscle, and Ala plays an important role in the regulation of blood glucose levels through the alanine-glucose cycle and gluconeogenesis in the liver [12]. In the three cases, the levels of Ala and Glx did not decrease throughout HD sessions after the first hour, but were maintained through homeostasis [13]. They are non-essential AA with biosynthesis that are closely interrelated with BCAAs, which could serve as amino group donors for the synthesis of Ala and Glx [14]. In this case report, growing levels of BCAA were suggested to provide a sufficient supply of amino groups for the synthesis of Ala and Glx.

As shown in Figs. 1C, 2C and 3C, IRI levels decreased due to diffusion, convection, and adsorption by membranes [15]. In contrast to the decrease 
in IRI levels, KB levels continuously increased. In this case report, increases of KB levels were more evident in the DM patients than in the non-DM patient, similar to BCAA behaviors. The former KB levels (Figs. 1C and $2 \mathrm{C}$ ) in the DM patients were elevated up to 600 to $1000 \mu \mathrm{M}$, far higher than the upper limit of the normal range $(100 \mu \mathrm{M})$ (arrows in right axes in Figs. 1C, $2 \mathrm{C}, 3 \mathrm{C})$. Such high values reflect starvation $[16,17]$. Circulating $\mathrm{KB}$ appears during starvation when the depletion of insulin induces free fatty acid release from adipose tissue, and $\beta$-oxidation accelerates in the liver $[16,17]$. The latter KB levels (Fig. 3C) in the nonDM patient showed slight increase in time-course behavior; however, these levels maintained within the normal range indicated to be not starvation during HD sessions.

In this case report, we investigated the circulating AA behaviors and profiles of insulin and ketone bodies during HD sessions in two DM patients and one non-DM patient to determine the effect of "HD starvation".

The comparison between HD starvation and conventional starvation (fasting starvation) is summarized in Table 2 [16-19]. A key similarity between the two is the extremely suppressed blood insulin level, whereas these differed in expression time: fasting starvation deteriorates gradually (20-36 h), and HD starvation deteriorates rapidly $(1 \mathrm{~h})$. The difference in the behavior of metabolites between the two states of starvation might reflect different liver statuses.

In the present three cases, IRI levels remained very low in the later stages of HD. This demonstrated that both DM and non-DM patients had only a small amount of endogenous insulin secretion stimulated by $100 \mathrm{mg} / \mathrm{dL}$ dialysate glucose. As described above, more prominent time-course changes in KB levels in patients with diabetes were coincident with insulin resistance often present in the groups [20].
To understand such unique pathophysiology of HD starvation, "difference of glucose transporter" expressed in different organ cell membranes should be considered. Glucose transporter GLUT4 (SLC2A4, solute carrier family 2 facilitated glucose transporter member 4) expressed in muscle cells and adipose tissue depends on insulin (insulin-sensitive organs), not on glucose [21, 22]. Under insulin depletion caused by HD session primarily via adsorption of insulin on the dialyzer membrane [15], GLUT4 cannot be translocated on the surface of muscle and adipose tissue, due to glucose poverty in these cells. Such cell starvation promotes adipose tissue lipolysis, which provides fatty acids to the liver through the bloodstream. Then, the liver accelerates $\beta$-oxidation for converting fatty acids into ketone bodies, amount of which flowed into blood with the consequence surpassing the continuous removal by HD (Figs. 1C, 2C, 3C). In contrast, the glucose transporter GLUT2 (SLC2A2, solute carrier family 2 facilitated glucose transporter member 2) expressed in the membranes of the liver and pancreas ( $\beta$-cell) are dependent on glucose (glucose-sensitive organs), not on insulin [23]. The relationship between glucose, insulin, and the organs is illustrated in Fig. 4. Because the PG level is almost constant during each HD session (Figs.1D, 2D and 3D), the liver and pancreas do not accelerate gluconeogenesis nor glucagon secretion [17] (Table 2).

In this context, the sharp increase in BCAA levels due to HD starvation (Figs. 1B, 2B, 3B) is interpreted as the distinct AA release from muscular cells via the autophagic procedure [24]. Basic adaptation of autophagy is to activate for nutrient starvation, the mechanism is critically sensitive to the levels of AA and insulin, and the effect of insulin is greater in muscle than liver [25].

Table 2 Comparison of time-course change in levels of metabolites between HD starvation and fasting starvation

\begin{tabular}{llll}
\hline Metabolite and related pathway & HD starvation & Fasting starvation & References regarding fasting starvation \\
\hline Blood glucose & $\rightarrow$ & $\downarrow$ & \\
Start of change & $1 \mathrm{~h}$ & $20-36 \mathrm{~h}$ & \\
Blood insulin & $\downarrow$ & $\downarrow$ & Owen [16] \\
Blood BCAA & $\uparrow$ & $\uparrow$ & Felig [18], Owen [16], Schauder [19] \\
Blood ketone bodies & $\uparrow$ & $\uparrow$ & Owen [16], Watford [17] \\
Hepatic glycogen & $\rightarrow$ & $\downarrow$ & Watford [17] \\
Blood alanine & $\rightarrow$ & $\downarrow$ & Felig [18] \\
Gluconeogenesis & $\rightarrow$ & $\uparrow$ & Felig [18], Watford [17]
\end{tabular}

The behaviors in lower three rows indicate difference of the liver status between two kind of starvation. In the right column, literatures referring to fasting starvation are depicted 


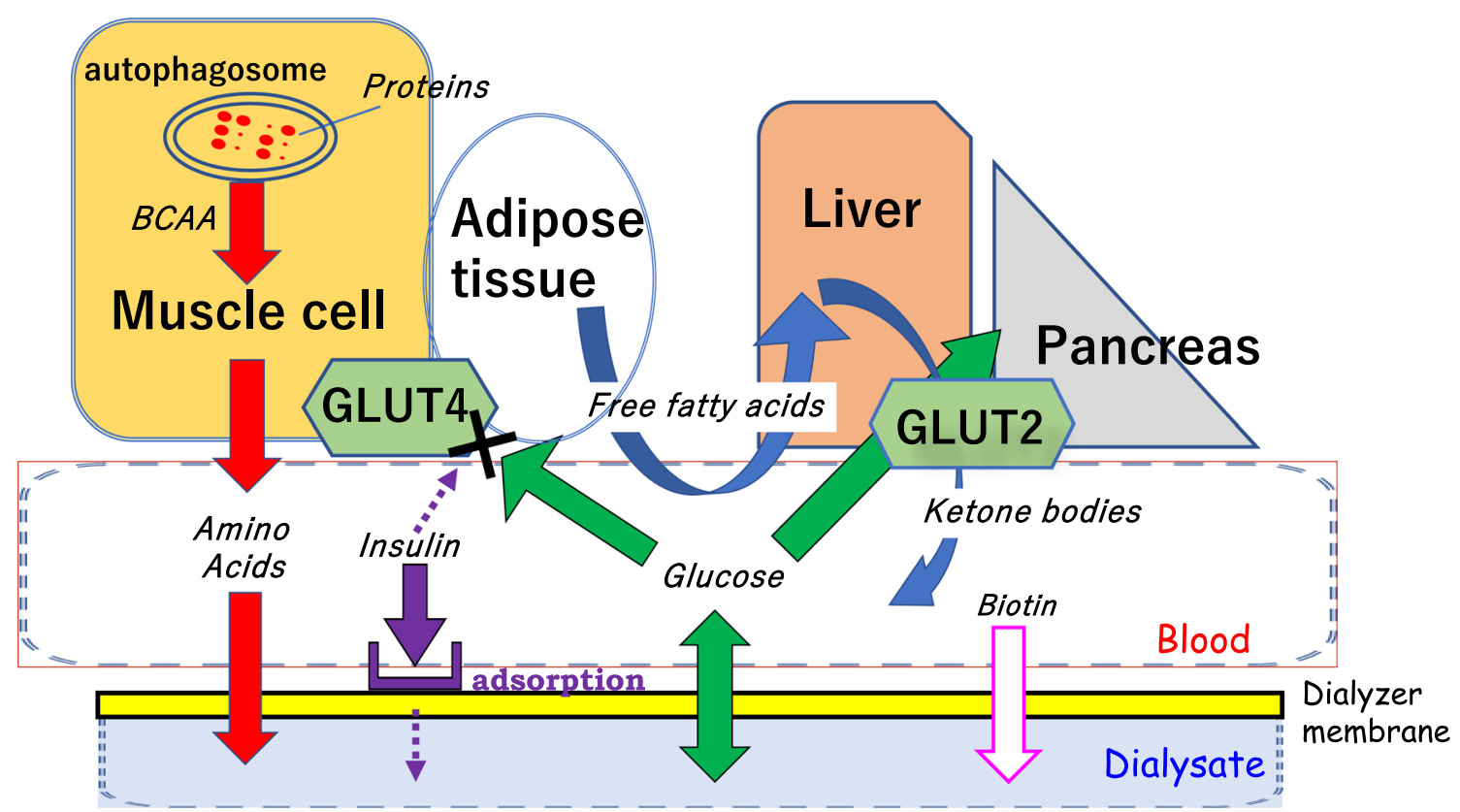

Fig. 4 The schema of HD starvation. Arrows are flows of metabolites: Dashed arrows indicate sparse little or no amount. Insulin is adsorbed by dialysis membrane. Amino acids depletion induced by HD session increases number of autophagosomes in muscle

The continuous increase in BCAA levels toward the end of the HD session suggests that there were no profound demands of the liver for BCAAs, suggesting that gluconeogenesis (transference of BCAA nitrogen to alanine, a substrate for gluconeogenesis) is not accelerated. In addition, "biotin deficiency" in HD patients [26, 27] could cooperatively suppress the gluconeogenesis during HD session, because biotin, a water-soluble vitamin, plays a key role as a coenzyme in reaction from pyruvate to oxaloacetic acid located at the start of the gluconeogenetic pathway.

The circulating levels of BCAAs are balanced by their appearance and disappearance in the blood. In this condition, an increase in supply and decrease in demand (consumption) of BCAA leads its circulating levels to elevation, and concomitantly, the amount missing of BCAA during $\mathrm{HD}$ into the dialysate is expected to increase. Therefore, an increase in plasma levels of BCAAs during HD is suspected to be harmful from the viewpoint of nutrient homeostasis.

Regarding HD starvation, an increase in plasma BCAA levels (Figs. 1B, 2B, 3B) supposed to be produced by the breakdown of muscle protein indicates disruption of homeostasis during HD in not only protein but also energy, because such high amounts of BCAAs are not available for energy generation due to the suppression of gluconeogenesis. Such suppression of gluconeogenesis could explain the time-course behavior of alanine levels (decrease initially and plateau thereafter). Except the initial removal during the sessions, alanine levels could be maintained by constant supply from plenty amounts of BCAA and cessation of consume for gluconeogenesis.

There are few studies regarding the change in AA concentration in the blood during HD treatment; representative studies are summarized in Table 3. The plasma levels of AAs were measured before and after each HD session, and the lack of decline in plasma EAA levels during the sessions has already been reported [2, 3, 8, 28-32]. However, the mechanism underlying EAA behavior remains unclear because plasma AA levels at intermediate time during the session are yet to be observed.

In summary, it is noted that the BCAA increase was triggered by IRI depletion and was accompanied by an elevation in KB levels. The synchronous time course changes in these metabolite levels represented disruption of homeostasis in protein and energy induced by HD starvation. In this case report, these behaviors were more evident in the DM patients than in the non-DM patient. This is the first case report to show the time course of the changes in circulating BCAAs in detail and clearly demonstrated an increase in BCAA levels at an early time in the sessions. The consequence was the dramatic increase in BCAA which suggested to induce skeletal protein breakdown. Present our finding is preliminary but novel and the larger study is warranted in near future. 


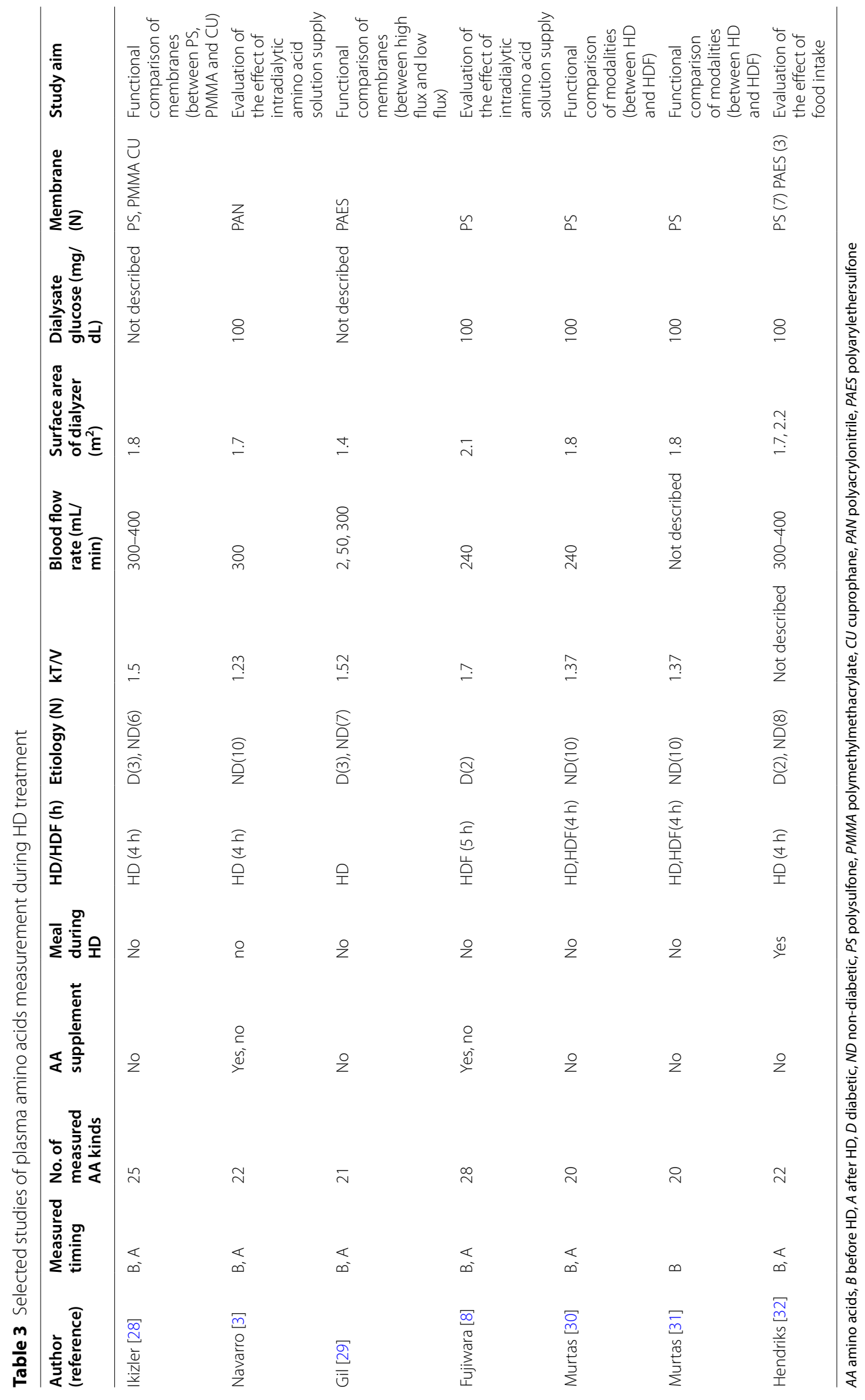




\section{Abbreviations}

HD: Hemodialysis; HDF: Hemodiafiltration; AA: Amino acid; BCAA: Branchedchain amino acid; EAA: Essential amino acid; NEAA: Non-essential amino acid; DM: Diabetes mellitus; IRI: Immunoreactive insulin; KB: Ketone bodies; Ala: Alanine; Glx: Glutamine, and glutamate; Leu: Leucine; Ile: Isoleucine; Val: Valine.

\section{Acknowledgements}

Not applicable.

\section{Authors' contributions}

MF and IA conceived and analyzed the data. MF wrote the draft of the manuscript. YI, YS, and HT revised the manuscript critically for important intellectual content. All authors read and approved the final manuscript.

\section{Funding}

This study received no specific funding.

\section{Availability of data and materials}

The data and materials were all included in the manuscript.

\section{Declarations}

\section{Ethics approval and consent to participate}

This study was approved by the Ethical Committee of Teikyo University (TU20-194), and informed consent was obtained from all the patients.

\section{Competing interests}

The authors declare no competing interests.

\section{Author details}

1 Department of Internal Medicine, Nephrology, Teikyo University Chiba Medical Center, 3426-3, Anesaki, Ichihara, Chiba 299-0111, Japan. ${ }^{2}$ Tohoku Institute for Management of Blood Pressure, 403, Station-Plaza Building, 13-18, Futsukamachi, Aobaku, Sendai, Miyagi 980-0802, Japan. ${ }^{3}$ Midorinosato Clinic, $27-2$ Mukaiyama-minami, Azahata, Kitahase, Iwanuma, Miyagi 989-2455, Japan.

\section{Received: 14 June 2021 Accepted: 22 December 2021}

Published online: 06 January 2022

\section{References}

1. Lim VS, Ikizler TA, Raj DSC, Flanigan MJ. Does hemodialysis increase protein breakdown? Dissociation between whole-body amino acid turnover and regional muscle kinetics. J Am Soc Nephrol. 2005;16:862-8.

2. Ikizler TA, Pupim LB, Brouillette JR, et al. Hemodialysis stimulates muscle and whole body protein loss and alters substrate oxidation. Am J Physiol Endocrinol Metab. 2002;282:E107-16.

3. Navarro JF, Mora C, León C, et al. Amino acid losses during hemodialysis with polyacrylonitrile membranes: effect of intradialytic amino acid supplementation on plasma amino acid concentrations and nutritional variables in nondiabetic patients. Am J Clin Nutr. 2000;71:765-73.

4. Mori K, Nishide K, Okuno S, et al. Impact of diabetes on sarcopenia and mortality in patients undergoing hemodialysis. BMC Nephrol. 2019;20:105.

5. Fujiwara M, Ando I, Takeuchi K, Oguma S, Sato H, Sekino H, et al. Metabolic responses during hemodialysis determined by quantitative $1 \mathrm{H}$ NMR spectroscopy. J Pharm Biomed Anal. 2015;111:159-62.

6. Fujiwara M, Ando I, Satoh K, et al. Biochemical evidence of cell starvation in diabetic hemodialysis patients. PLoS ONE. 2018;13:e0204406.

7. Yamamoto H, Kondo K, Tanaka T, et al. Reference intervals for plasmafree amino acid in a Japanese population. Anna Clin Biochem. 2016;53:357-64

8. Fujiwara M, Ando I, Nemoto T, et al. Amino acid kinetics in diabetic patients during hemodiafiltration performed under intradialytic parenteral amino acid nutrition: a preliminary study. J Jpn Soc Dial Ther. 2019;52:457-62

9. Ando I, Hirose T, Nemoto T, et al. Quantification of molecule in ${ }^{1} \mathrm{H}-\mathrm{NMR}$ metabolomics with formate as a concentration standard. J Toxicol Sci. 2010;35(2):253-6.
10. Ando I, Takeuchi K, Oguma S, et al. ${ }^{1} \mathrm{H}$ NMR spectroscopic quantification of plasma metabolites in dialysate during hemodialysis. Magn Reson Med Sci. 2013:12(2):129-35.

11. Iwase H, Kobayashi M, Nakajima M, Takatori T. The ratio of insulin to C-peptide can be used to make a forensic diagnosis of exogeneous insulin overdosage. Foren Sci Intern. 2001;115:123-7.

12. Raj DSC, Welbourne T, Dominic EA, et al. Glutamine kinetics and protein turnover in end-stage renal disease. Am J Physiol Endcronol Metab. 2005:288:E37-46.

13. Bröer S, Bröer A. Amino acid homeostasis and signaling in mammalian cells and organisms. Biochem J. 2017:474:66.

14. Harper AE, Miller RH, Block KP. Branched-chain amino acid metabolism. Annu Rev Nutr. 1984:4:409-545.

15. Abe M, Kalantar-Zadeh K. Haemodialysis-induced hypoglycaemia and glycaemic disarrays. Nat Rev Nephrol. 2015;11:302-14.

16. Owen OE, Felig P, Morgan AP, Wahren J, Cahil GF. Liver and kidney metabolism during prolonged starvation. J Clin Invest. 1969;48:574-83.

17. Watford M: Starvation. Metabolic changes. In: eLS. Wiley: Chichester. 2015.

18. Felig P, Owen E, Wahren J, Cahill DF Jr. Amino acid metabolism during prolonged starvation. J Cli Invest. 1969;48:584-94.

19. Schauder $P$, Herbertz L, Langenbeck U. Serum branched chain amino and keto acid response to fasting in humans. Metabolism. 1985;34:58-61.

20. Rahhal MN, Ghraibeh NE, Rahimi L, Ismal-Beigi F. Disturbances in insulinglucose metabolism in patients with advanced renal disease with and without diabetes. J Clin Endocrinol Metab. 2019;104:4949-66.

21. Bell G, Kayano T, Buse J, et al. Molecular biology of mammalian glucose transporters. Diabetes Care. 1990;13:198-208.

22. Watson RT, Kanzaki M, Pessin JE. Regulated membrane trafficking of the insulin-responsive glucose transporter 4 in adipocytes. Endcrine Rev. 2004; $25: 177-204$

23. Thorens B. Glucose transporters in the regulation of intestinal, renal, and liver glucose fluxes. Am J Physiol. 1996;270:G541-53.

24. Boya P, Gonzalez-Polo RA, Casares N, Perfettini JL, Dessen P, Larochette $\mathrm{N}$, et al. Inhibition of macroautophagy triggers apoptosis. Mol Cell Biol. 2005:25:1025-40.

25. Naito T, Kuma A, Mizushima N. Differential contribution of insulin and amino acids to the mTORC 1-autophagy pathway in liver and muscle. J Biol Chem. 2013:288:21074-81.

26. Oguma S, Ando I, Hirose T, et al. Biotin ameliorates muscle cramps of hemodialysis patients: a prospective trial. Tohoku J Exp Med. 2012;227:217-23

27. Fujiwara M, Ando I, Yagi S, et al. Plasma levels of biotin metabolites are elevated in hemodialysis patients with cramps. Tohoku J Exp Med. 2016;239(4):263-7.

28. Ikizler TA, Flakoll PL, Parker RA, Hakim RM. Amino acid and albumin losses during hemodialysis. Kidney Int. 1994;46:830-7.

29. Gil HW, Yang JOY, Lee EM, Choi JS, Hong SY. The effect of dialysis membrane flux on amino acid loss in hemodialysis patients. J Korean Med Sci. 2007:22:598-603.

30. Murtas S, Aquilani R, Deiana ML, et al. Differences in amino acid loss between high-efficiency hemodialysis and postdilution and predilution hemodiafiltration using high convection volume exchange-a new metabolic scenario? A pilot study. J Ren Nutri. 2019:29:126-35.

31. Murtas S, Aquilani R, ladarola P, et al. Differences and effects of metabolic fate of individual amino acid loss in high-efficiency hemodialysis and hemodiafiltration. J Ren Nutri. 2020;30:440-51.

32. Hendriks FK, Smeets JSJ, Broers NJH, et al. End-stage renal disease patients lose a substantial amount of amino acids during hemodialysis. J Nutr. 2020;31:1160-6.

\section{Publisher's Note}

Springer Nature remains neutral with regard to jurisdictional claims in published maps and institutional affiliations. 